\title{
O impacto das condições de vida e da educação sobre a incidência de tuberculose no rasil
}

Evandro Camargos Teixeira ${ }^{1}$

Jaqueline Severino da Costa ${ }^{2}$

Resumo: A Tuberculose pode ser considerada uma “doença de países pobres", dado que ela tem avançado em países com baixo nível de renda e educação, como é o caso do Brasil. Dessa forma, o objetivo do presente trabalho é analisar os determinantes socio econômicos da Tuberculose, enfatizando o nível educacional e as condições de vida. Para alcançar tal objetivo, foi utilizado um modelo Probit, tendo como variável dependente a condição de estar ou não contaminado pela doença. Os resultados demonstram que um indivíduo do sexo masculino, negro, idoso, com baixo nível de escolaridade, e que vive em péssimas condições de vida na zona urbana do Sul do país apresenta a maior probabilidade de ser infectado pela tuberculose.

Palavras-chave: tuberculose; educação; condições de vida; economia da saúde. (UFOP) e doutorando em Economia Aplicada pela Escola Superior de Agricultura Luiz de Queiroz (ESALQ) / campus Universidade de São Paulo (USP). E-mail para contato: evandro@icsa.ufop.br

2 Pesquisadora do Instituto Jones dos Santos Neves/Vitória - ES e doutoranda em Economia Aplicada pela Escola Superior de Agricultura Luiz de Queiroz (ESALQ)/ campus Universidade de São Paulo (USP). E-mail para contato: jaqueline.s.costa@hotmail.com. 


\title{
The Impact of Living and Education on the Incidence of Tuberculosis in Brazil
}

\begin{abstract}
The Tuberculosis can be considered a "disease of poor countries," since it has advanced in countries with low income and education, as is the case in Brazil. Thus, the purpose of this study is to analyze the socio-economic aspects of tuberculosis, emphasizing the educational level and living conditions. To achieve this, we used a Probit model, the dependent variable condition is or is not contaminated by the disease. The results show that a indiviuo male, black, elderly, low education level, and living in horrible living conditions in urban areas in the South has the highest probability of being infected by tuberculosis.
\end{abstract}

Keywords: tuberculosis; education; life conditions; health economics.

JEL: I10

\section{Introdução}

Segundo o relatório da OMS 2003 - "Controle Global da Tuberculose: Vigilância, Planejamento, Financiamento" - a incidência mundial da tuberculose vem crescendo em aproximadamente $0,4 \%$ ao ano. Portanto, essa doença ainda consiste em um grave problema de saúde pública, principalmente na Ásia, na África e na América do Sul. A incidência da tuberculose está altamente relacionada a questões socio econômicas, ou seja, está associada à pobreza e à má distribuição de renda.

De acordo com a Organização Mundial da Saúde (OMS), em 2005 cerca de 1/3 da população mundial, ou seja, 2 bilhões de pessoas, foi acometido pela tuberculose ${ }^{3}-$ Mycobacterium tuberculosis (tuberculose). Atualmente, cerca de 100 milhões de pessoas, em média têm sido infectadas por ano. Existe ainda uma previsão de que 8 milhões de pessoas desenvolverão a doença e 2 milhões morrerão a cada ano. Cabe ressaltar que $80 \%$ de todos os casos ocorreram em 22 países, particularmente em países do continente Africano e Asiático.

3 A tuberculose é uma doença infecciosa que atinge principalmente os pulmões, mas ela pode atingir outros órgãos como gânglios, rins, ossos, meninges ou outros locais do organismo. Em 1882, Robert Koch descobre o agente causador da TUBERCULOSE, o bacilo de Koch. A descoberta foi importante para conhecer a doença e impulsionou várias tentativas de controle e tratamento da enfermidade. No final do século XIX, a doença passou a estar relacionada às condições precárias de vida dos indivíduos residentes em habitações pequenas para o número de moradores, péssimas condições alimentares e falta de higiene. E nesta época o tratamento higieno-dietético era o mais importante, pois acreditava-se que a cura do doente acontecia com boa alimentação, repouso e lugares com podia-se viver no clima das montanhas. No século XX, a descoberta de medicamentos específicos nos anos 1940 permitiu uma diminuição rápida dos índices de mortalidade pela doença. No entanto, com o surgimento e a propagação da Síndrome da lmunodeficiência Adquirida (AIDS), o aumento da pobreza, aceleração da urbanização e a ausência de controle social vem impondo dificuldades no controle da doença. 
Em contrapartida, de acordo com OMS (2005), a Europa concentra apenas 5\% dos casos de tuberculose, isto é, no continente europeu a incidência, a internação e a mortalidade são relativamente baixas. No entanto, alguns países europeus apresentam incidência da doença nos níveis da África e da Ásia, devido principalmente a elevada resistência da tuberculose aos medicamentos.

Analisando a incidência da tuberculose no Brasil, segundo dados da OMS (2005), concluí-se que o país está entre os maiores responsáveis pelo total de casos da doença no mundo ${ }^{4}$. Estima-se que no Brasil há uma prevalência de 50 milhões de infectados com cerca de 111 .0oo casos novos por ano, o que corresponde a um coeficiente de incidência de 47/ 100.000 habitantes e 6.000 óbitos ocorrendo anualmente em virtude da doença.

Dado esse grave impacto no Brasil, o objetivo do presente trabalho consiste em apontar os principais determinantes da tuberculose no Brasil, com ênfase nos efeitos da educação e das condições de vida. Para atingir tal objetivo, o trabalho está dividido em mais cinco seções, além dessa introdução. Na segunda seção, será apresenta a revisão de literatura que tange o tema do trabalho. Na seção seguinte, é apresentado o modelo empírico. Na quarta seção, são analisadas as estatísticas descritivas. Na seção subseqüente são apresentados e discutidos os resultados das equações estimadas segundo o gênero. Por fim, são apresentadas as considerações finais.

\section{Determinantes da tuberculose: uma revisão de literatu- ra}

De acordo com Rieder (2001), a incidência de tuberculose apresenta uma relação direta com a conjuntura socio econômica. Porém, a literatura que concerne à análise dos determinantes da tuberculose em todo o mundo é relativamente incipiente. A maioria dos trabalhos realizados até o momento se baseia em análises ecológicas, tendo como base censos populacionais ou códigos postais.

Os primeiros estudos foram conduzidos no início do século XX. D’Arcy Hart (1937) resumiu três destes estudos realizados em Trondheim (na Noruega), Eidelberg (na Alemanha) e Hué (no Vietnam). Segundo o autor, as diferenças na prevalência específica por idade, entre os segmentos mais ricos e os mais pobres da população, são substanciais.

Após esse estudo, a inter-relação entre pobreza e tuberculose ganhou força

4 O país ocupa $15^{\circ}$ posição entre os 22 países. A frente do Brasil, Índia, China, Indonésia, Nigéria, Bangladesh, Etiópia, Filipinas, Paquistão, África do Sul, Rússia, Congo, Quênia, Vietnã e Tanzânia compõe os países com maior incidência de casos de tuberculose. 
na literatura. Kuemmerer e Comstock (1967) examinaram a variação da prevalência da infecção entre estudantes universitários nos Estados Unidos, em função do nível educacional dos pais e das características das suas habitações. Os autores concluíram que a sobrelotação era um determinante importante das grandes incidências de tuberculose.

A hipótese de relação direta, citada no parágrafo anterior, entre a incidência de tuberculose e sobrelotação das moradias apresenta uma explicação interessante. O baixo nível socio econômico tende a dar origem a sobrelotações habitacionais. Com a ocorrência de um caso da doença, haverá um aumento na transmissão do bacilo e consequentemente uma elevação da incidência de tuberculose. Essa densidade populacional difere entre as regiões rurais e regiões urbanas, segundo o "Ministry of Health and Social Affairs, Korean Institute of Tuberculosis” (1966). Os autores verificaram que, na Coreia, a incidência de tubeculose era maior nas áreas urbanas do que nas rurais.

Além disso, como salientado por Bergner (1968), a pobreza pode também reduzir o acesso aos serviços de saúde, prolongando o período de contágio dos doentes e aumentando o risco de infecção entre as pessoas mais próximas. Isso vem se confirmando nos últimos anos, como demonstrado nos estudos de Enarson, Wang, e Dirks (1989); e Cantwell et al. (1994). Tupasi et al (2000) compararam a incidência de tuberculose entre a população mais pobre e o restante da população residente em regiões metropolitanas das Filipinas. Os autores concluíram que a tuberculose afeta mais a população urbana pobre do que a população urbana em geral. Isso seria explicado, principalmente, pela excessiva migração de indivíduos da zona rural para as grandes metrópoles em busca de melhores condições de vida. Essas pessoas se aglomeram, geralmente, nos lugares mais pobres, vivendo em péssimas condições de vida. Muitos estudos têm explorado a infecção por tuberculose relacionada ao sexo e à idade da vítima. Groth-Petersen, Knudsen e Wilbek (1959) e o National Tuberculosis Institute Bangalore (1974) demonstraram a prevalência da infecção específica por idade, agrupada por sexo, na Dinamarca e na Índia, respectivamente. Em ambos os países, a incidência de tuberculose se eleva após a infância, e aumenta mais rapidamente entre os homens.

Nesse sentido, Holmes, Hausler e Null (1998) chegaram à conclusão que na maioria dos países a incidência de tuberculose entre homens e mulheres é similar até a adolescência. Após os 15 anos, a taxa entre homens excede a de mulheres. Além disso, os autores revelaram uma outra relação: indivíduos que são HIV positivo têm um risco de contrair tuberculose de duas a cem vezes maior que os demais indivíduos.

Além dessas análises, em um estudo realizado em um hospital público de Atlanta (Eua) entre outubro de 1993 e dezembro de 1994, Bock, McGowan e Blumberg (1998) confirmaram a hipótese de que os homens são mais afetados pela doença e relataram uma outra característica importante no que tange a etnia das vítimas: $89 \%$ são negros com idade média de 40 anos. 
Analisando-se essa relação entre a etnia, gênero e idade, Martinez, Small e Behr (2000) analisaram um grupo de indivíduos com tuberculose entre os anos de 1991 e 1996 em São Francisco (EUA). Os autores concluíram que a taxa média anual de incidência foi de 57,3/100.00o habitantes para homens e 26,8/100.00o habitantes para mulheres, sendo que até os 24 anos a incidência foi similar. A partir dos 25 anos, o risco para os homens é, geralmente, de duas a três vezes maior. Analisando-se a questão étnica, concluiu-se que os negros são mais afetados pela doença, sendo que essa diferença é mais elevada na faixa etária de 25-64 anos.

Como salientado anteriormente, os estudos que relacionam a incidência de tuberculose às condições socio econômicas são incipientes em todo mundo. Porém, no Brasil, tais estudos são mais escassos ainda. Vicentin, Santo e Carvalho (2002) utilizaram um modelo ecológico para analisar a relação entre o Coeficiente de Mortalidade por Tuberculose (CMTB) com alguns indicadores sociais em diferentes regiões administrativas e áreas de planejamento no município do Rio de Janeiro no ano de 1991. Os autores concluíram que os coeficientes mais elevados de mortalidade por tuberculose são encontrados nas regiões administrativas onde predominam populações de baixa renda. Entre os indicadores sociais, o mais sensível à CMTB foi o número de cômodos por município, seguido da área média por domicílio.

Santos et al. (2007) estabeleceram uma relação entre a incidência de tuberculose e as características socio econômicas do município de São José do Rio Preto (SP) entre os anos de 1998 e 2004. O estudo confirmou a hipótese de que existe uma forte relação entre as condições de vida e o padrão de mortalidade por tuberculose. Dentre as variáveis socio econômicas identificadas na análise, os anos de instrução/analfabetismo, a renda dos responsáveis por domicilio, e os habitantes por domicilio foram as que mais impactaram na incidência da doença.

Diante das variáveis consideradas relevantes pela literatura e dada a escassez de trabalhos que analisam os determinantes socio econômicos da tuberculose, o presente trabalho propõe uma outra abordagem, utilizando o modelo empírico apresentado a seguir.

\section{Modelo empírico}

\subsection{Dados utilizados}

No presente estudo utilizou-se para as estimações os dados da Pesquisa Nacional por Amostra de Domicílio (PNAD), em especial o seu suplemento 
sobre saúde de 2003. A PNAD é uma pesquisa realizada pelo Instituto Brasileiro de Geografia e Estatística (IBGE) desde 1967, cujos objetivos são buscar informações que podem apontar diversas características socioeconômicas da população brasileira, dentre elas aquelas relacionadas à educação, renda, salários e habitação. A forma como é estabelecida a pesquisa permite que os resultados sejam expandidos para todo o país, bem como para suas regiões, estados e áreas urbanas.

A PNAD utilizada inclui 384.585 mil indivíduos das regiões Norte, Nordeste, Sudeste, Sul, Centro-Oeste da área urbana e rural de todo o Brasil. A pesquisa considera informações sobre as características dos indivíduos como anos de estudo, sexo, idade, domicílio e cor.

Cabe ressaltar que apesar desta base de dados possuir algumas limitações, a análise empírica da relação entre tuberculose e pobreza ainda assim oferece grandes vantagens:

- $\quad$ grande número de observações;

- possibilidade de controlar diversos fatores, que podem afetar a probabilidade de um indivíduo ser acometido pela doença;

- poder de controle do erro de medida, quando são utilizados dados de saúde autoreportada, ou seja, quando a pergunta é feita ao próprio indivíduo se ele tem tuberculose diagnosticada por um médico;

- permite a redução de um viés de seleção de amostra, pois a mesma foi composta de indivíduos acometidos ou não pela doença. Assim, pode-se observar os efeitos das variáveis de interesse, buscando estimar quais características são mais determinantes para o individuo ser acometido pela tuberculose.

Nesse sentido, foram estimadas quais as características mais determinantes para o individuo ser infectado pela tuberculose. No entanto, para o presente trabalho foi realizada uma filtragem na amostra para diminuir alguns problemas que poderiam ser irrelevantes ao estudo. Assim, optou-se por excluir todos os indivíduos que não responderam à pergunta sobre a presença ou não de alguma das doenças crônicas consideradas na pesquisa.

\subsection{Modelo utilizado}

A tuberculose é um estado e não uma doença permanente, porém seu tratamento é longo, com duração média de 6 meses. A tuberculose pertence ao grupo das 11 doenças crônicas consideradas importantes pelo suplemento especial de saúde da PNAD de 2003. Nesse sentido, se um indivíduo estiver 
em contato direto com um doente infectado pela tuberculose pulmonar, pode acabar sendo contaminado.

A estimação utilizada no presente trabalho foi realizada pelo método da máxima verossimilhança utilizando modelos probit. O modelo probit é apresentado abaixo:

$$
\operatorname{Prob}(Y=1 \mid x)=\int_{-c}^{\prime \ell} \phi(t) d t=\phi\left(x, \beta^{\prime}\right)
$$

Assim, a variável considerada como dependente no modelo é binária, ou seja, assume 1 , caso o individuo tenha tuberculose (tuberculose $=1$ ), e 0 , caso contrário (tuberculose $=0$ ). A partir da equação 1 estimam-se os efeitos marginais que serão considerados para as análises a seguir:

$$
\frac{\partial E[y \mid x]}{\partial x}=\phi\left(x^{\prime}, \beta\right) \beta
$$

Nesse caso, os efeitos marginais representam, por exemplo, o quanto uma mudança na variável educação pode afetar a probabilidade de um indivíduo estar com tuberculose. Os efeitos marginais (a variação percentual da probabilidade do evento ocorrer quando uma variável independente é modificada) são calculados através dos coeficientes estimados $\beta$ 's:

Os fatores que contribuem para a proliferação da tuberculose estão relacionados a grandes concentrações populacionais e péssimas condições socio econômicas. Cabe ressaltar que estas condições podem ser expressas de forma indiretamente por algumas das variáveis explicativas, que serão apresentadas a seguir, como educação, condição do domicílio (urbano ou rural), idade do indivíduo, tamanho do domicilio, condições sanitárias, cor e região geográfica.

Conforme Santos e Kassouf (2007), a variável anos de estudo aponta que um ano mais de estudo no ensino fundamental provavelmente tem impacto diferente de um ano a mais no ensino médio ou superior. Por isso, torna-se importante dividir a variável anos de estudo em categorias (níveis educacionais). Nesse sentido, neste trabalho optou-se por utilizar níveis de educação para captar os diversos impactos que estas podem ter sobre a probabilidade de um indivíduo ser acometido pela tuberculose. Assim, a variável anos de estudo foi definida em 5 níveis: EDU1 (indivíduos que apresentam nenhuma instrução ou até 1 ano de estudo), EDU2 (indivíduos que possuem entre 2 e 5 anos de estudo), EDU3 (indivíduos que possuem entre 6 e 9 anos de estudo), EDU4 (indivíduos que possuem entre 10 e 12 anos de estudo), e EDU5 (indivíduos que possuem 13 ou mais anos de estudo). 
As demais variáveis incluídas no modelo para controle são: URBAN (se o indivíduo reside na área urbana); SUL, SUD, NOR, NORD e CENTROESTE (qual região geográfica o mesmo pertence); IDADE1 (idade entre o e 14 anos); IDADE2 (idade entre 15 e 60 anos); IDADE3 (idade superior a 60 anos); COR1 (se o indivíduo possui cor branca); COR2 (se o indivíduo possui cor parda); COR3 (se o indivíduo possui cor negra); DOM (tamanho do domicílio, que é representado pelo número de cômodos); e SAN (condições sanitárias apresentadas pelo indivíduo). As variáveis do modelo foram transformadas em binárias para controlar possíveis diferenças na probabilidade de um indivíduo ser vitimizado pela doença. Assim, no modelo utilizado algumas variáveis foram omitidas (CENTROESTE, IDADE1, EDU1, NEGRO), de modo a serem utilizadas como base. Cabe sublinhar que a análise é dividida em duas equações, segundo o gênero do indivíduo.

De acordo com a análise de alguns estudos empíricos já realizados, esperase que os efeitos sejam positivos para IDADE2, IDADE3 e URBAN, ou seja, devido à elevação na idade e pelo fato do indivíduo residir na área urbana, tem-se um aumento na probabilidade de se contrair tuberculose.

Com relação às variáveis de educação (EDU1, EDU2, EDU3, EDU4 e EDU5), tamanho do domicílio (DOM), e condições sanitárias (SAN) esperam-se efeitos negativos. Assim, à medida que se eleva o nível de educação, aumenta o tamanho do domicílio e melhoram as condições sanitárias de uma residência; o que diminui a probabilidade do indivíduo ser acometido pela tuberculose. Contudo, para as variáveis regiões geográficas e cor não é realizada nenhuma expectativa quanto aos efeitos a serem verificados.

Cabe ressaltar que a variável de maior importância no trabalho é "anos de estudo" (níveis de educação), pois esta além de ser a única que pode ser controlada por políticas públicas, pode ainda captar de forma indireta as condições socio econômicas de um indivíduo. Portanto, o modelo utilizado é bem simples, sendo que a probabilidade de um indivíduo ser acometido pela tuberculose depende do nível de instrução, de algumas características individuais e das condições de vida dos indivíduos.

A Tabela 1 aponta a média e o desvio padrão das variáveis a serem consideradas nas equações estimadas para homens e mulheres. Observa-se que dentro da amostra selecionada para o modelo, $0,17 \%$ dos homens tinham tuberculose, enquanto $0,14 \%$ das mulheres apresentavam a doença. Percebe-se que os homens são de maneira geral mais afetados pela doença do que as mulheres. 
TABELA 1 . MÉDIA E DESVIO PADRÃO DAS VARIÁVEIS

\begin{tabular}{clllll}
\hline \multirow{2}{*}{ Variáveis } & \multicolumn{1}{c}{ Descrição } & \multicolumn{2}{c}{ Homens } & \multicolumn{2}{c}{ Mulheres } \\
& & Média & $\begin{array}{c}\text { Desvio } \\
\text { Padrão }\end{array}$ & Média & $\begin{array}{c}\text { Desvio } \\
\text { Padrão }\end{array}$ \\
\hline TUBERCULOSE & $=1$ se tem tuberculose & 0,0017 & 0,041 & 0,0014 & 0,037 \\
EDU1 & $=1$ se tem entre 0 e 4 anos de estudo & 0,248 & 0,432 & 0,232 & 0,422 \\
EDU2 & $=1$ se tem entre 2 e 5 anos de estudo & 0,267 & 0,442 & 0,250 & 0,433 \\
EDU3 & $=1$ se tem entre 6 e 9 anos de estudo & 0,237 & 0,425 & 0,230 & 0,421 \\
EDU4 & $=1$ se tem entre 10 e 12 anos de estudo & 0,178 & 0,382 & 0,201 & 0,401 \\
EDU5 & $=1$ se tem 13 ou mais anos de estudo & 0,068 & 0,251 & 0,083 & 0,276 \\
IDADE & $=$ idade do indivíduo & 28,38 & 19,60 & 30,15 & 20,31 \\
IDADE1 & $=1$ com idade entre 0 e 14 anos & 0,289 & 0,453 & 0,264 & 0,441 \\
IDADE2 & $=1$ com idade entre 15 e 60 anos & 0,628 & 0,483 & 0,635 & 0,481 \\
IDADE3 & $=1$ com idade superior a 60 anos & 0,081 & 0,274 & 0,099 & 0,299 \\
COR1 & $=1$ se a cor é branca & 0,465 & 0,498 & 0,448 & 0,499 \\
COR2 & $=1$ se a cor é parda & 0,469 & 0,499 & 0,443 & 0,496 \\
COR3 & $=1$ se a cor for negra & 0,065 & 0,247 & 0,061 & 0,239 \\
URBANO & $=1$ se vive na zona urbana & 0,846 & 0,360 & 0,866 & 0,339 \\
DOM & Tamanho do domićlio & 5,865 & 2,249 & 5,929 & 2,267 \\
SAN & Condições sanitárias & 0,931 & 0,253 & 0,940 & 0,236 \\
NORT & $=1$ se reside na região Norte & 0,097 & 0,296 & 0,096 & 0,295 \\
NORD & $=1$ se reside na região Nordeste & 0,325 & 0,468 & 0,325 & 0,468 \\
SUD & $=1$ se reside na região Sudeste & 0,294 & 0,455 & 0,297 & 0,457 \\
SUL & $=1$ se reside na região Sul & 0,156 & 0,363 & 0,157 & 0,363 \\
CENTROESTE & $=1$ se reside na região Centro-Oeste & 0,125 & 0,331 & 0,122 & 0,328 \\
\hline
\end{tabular}

FONTE: elaborado pelos autores com base nos dados da PNAD (2003)

A variável educação apresentada a partir da Tabela 1 aponta que grande parte dos homens concentra-se entre os níveis de escolaridade mais baixos como EDU1, EDU2 e EDU3, ou seja, 24,8\%, 26,6\% e 23,7\%, respectivamente. Em relação às mulheres, 23,2\%, 25,0\% e 23,0\% das mesmas pertencem aos níveis de educação EDU1, EDU2 e EDU3, respectivamente. À medida que são analisados os níveis de educação mais elevados, percebe-se que há um predomínio dos indivíduos do sexo feminino. Assim, nota-se que 20,1\% e 8,3\% das mulheres apresentam níveis de educação EDU4 e EDU5 (os mais elevados) respectivamente. Já entre os homens, 17,8\% e 6,08\% possuem o nível mais elevado de educação. Contudo, quando são considerados tanto homens quanto mulheres o nível de educação em que há uma concentração maior de indivíduos é EDU2.

Para as demais variáveis, como idade média dos indivíduos, percebe-se que a idade média entre as mulheres (30,15 anos) é maior do que entre os homens (28,4 anos), conforme a Tabela 1. Quando se divide a idade em classes, percebe-se que há um predomínio de mulheres entre as faixas de IDADE2 e IDADE3, ou seja, a maioria das são adultas e idosas. Porém, na faixa de IDADE1, constituída por crianças e jovens, observa-se que há um predomínio de indivíduos do sexo masculino.

De acordo com a Tabela 1, a quantidade de mulheres de cor branca $(48,9 \%)$ é superior ao número de homens (46,6\%), enquanto que entre os homens há um predomínio da cor parda (46,2\%) em relação às mulheres (44,3\%). A variável DOM aponta que o tamanho dos domicílios ocupados pelos homens são, em média, menores em relação àqueles ocupados pelas mulheres. Essa relação 
é importante, pois quanto menor o tamanho do domicilio, ou seja, quanto maior o número de pessoas concentradas nos domicílios, mais elevadas são as probabilidade de um individuo ser infectado pela tuberculose.

Analisando-se as condições sanitárias, tendo como proxy o fato de um domicílio ter banheiro ou sanitário, percebe-se que as mulheres vivem em melhores condições do que os homens.

De acordo com a Tabela 1, percebe-se que há uma concentração da população na zona urbana, ou seja, em média $85 \%$ dos indivíduos residem nas cidades. Cabe destacar que o percentual de mulheres que vivem na zona urbana supera quantidade de homens: $86,6 \%$ contra $84,6 \%$.

Em termos de região geográfica, observa-se que não há um predomínio de um sexo em relação ao outro, ou seja, há uma distribuição eqUitativa entre homens e mulheres.

\section{Análise descritiva dos dados}

Esta seção tem o objetivo de analisar as variáveis que determinam a incidência de tuberculose segundo gênero, o que será feito na próxima seção.

Os resultados da Tabela 2 permitem apontar que do total da amostra, $48,74 \%$ são homens e que 51,26\% são mulheres, o que demonstra um predomínio de mulheres. Quando são analisados os indivíduos que não foram infectados pela tuberculose, observa-se que há um predomínio de mulheres em relação aos homens, $51,27 \%$ e $48,73 \%$, respectivamente. Porém, quando se considera os indivíduos que possuem tuberculose, observa-se que há um predomínio da doença entre os homens, 53,9\% do total. Portanto, percebe-se que de forma geral a doença é mais comum entre homens do que entre mulheres.

TABELA 2. PORCENTAGEM DE HOMENS E MULHERES DO TOTAL DAAMOSTRA E CONDICIONAL A DOENÇA TUBERCULOSE

\begin{tabular}{lccc}
\hline Sexo & Total da amostra (\%) & $\begin{array}{c}\text { Pessoas sem T } \\
\text { tuberculose }(\%)\end{array}$ & $\begin{array}{c}\text { Pessoas com tuberculose } \\
(\%)\end{array}$ \\
\hline Masculino & 48,74 & 48,73 & 53,90 \\
Feminino & 51,26 & 51,27 & 46,10 \\
\hline
\end{tabular}

FONTE: elaborado pelos autores com base nos dados da PNAD (2003)

Segundo dados da Tabela 3, observa-se que a média de anos de estudos para homens que não foram vitimas da TUBERCULOSE é de 6,1, enquanto que para as mulheres a média é de 6,5 anos. Isso prova que, em média, as mulheres estudam mais que os homens. Para os indivíduos que são acometidos pela doença observa-se que os homens continuam em média com um número menor de anos de estudos, 5,8 anos, contra 6,2 anos das mulheres. 
TABELA 3. ANOS DE ESTUDO MÉDIO, IDADE MÉDIA E NÚMERO MÉDIO PESSOAS POR CÔMODO CONDICIONADA A DOENÇA TUBERCULOSE

\begin{tabular}{lccc}
\hline Variáveis & Gênero & Pessoas sem tuberculose & Pessoas com tuberculose \\
\hline \multirow{2}{*}{ Anos de estudo } & Masculino & 6,1 & 5,8 \\
& Feminino & 6,5 & 6,2 \\
\multirow{2}{*}{ Idade } & Masculino & 28,3 & 44,7 \\
\multirow{2}{*}{ Cômodo } & Feminino & 30,1 & 44,4 \\
& Masculino & 5,86 & 5,43 \\
& Feminino & 5,93 & 5,42 \\
\hline
\end{tabular}

FONTE: elaborado pelos autores com base nos dados da PNAD (2003)

Assim, pode-se inferir, a partir da Tabela 3, que, em média, indivíduos com mais anos de estudo possuem menos risco de contraírem a doença, o que pode ser verificado tanto para homens como para as mulheres.

Conforme a Tabela 3, a idade média, para homens que não foram infectados pela tuberculose é menor quando comparada as mulheres. Em média, os homens que não foram contaminados pela tuberculose têm 28,3 anos, enquanto as mulheres têm 30,1 anos. Quando se considera indivíduos acometidos pela tuberculose, observa-se que a média de idade aumenta tanto para homens quanto para mulheres. Porém, um fato a ser destacado é que a média de idade para indivíduos com tuberculose é maior entre homens do que entre mulheres, 44,7 e 44,4 anos, respectivamente. Desta forma, percebe-se que a doença atinge indivíduos com faixa etária mais elevada, ou seja, à medida que a idade avança maiores são as chances de um indivíduo contrair a doença.

A variável "tamanho do domicílio" é muito importante na explicação da incidência de tuberculose. Assim, de acordo com a Tabela 3, percebe-se que entre os indivíduos que não são acometidos pela tuberculose o tamanho do domicílio é maior, quando comparado aos domicílios dos indivíduos que tem tuberculose. Para as mulheres infectadas pela tuberculose, o tamanho dos domicílios em média, é maior em relação aos homens. No entanto, quando são analisados os indivíduos com tuberculose percebe-se que o tamanho dos domicílios se equivale, ou seja, o numero de cômodos em média é igual para ambos os sexos.

De acordo com a Tabela 4, observa-se que a maior parte dos indivíduos (homens e mulheres) reside na área urbana. Quando se compara homens e mulheres, que moram na zona urbana, e que foram infectados pela tuberculose, percebe-se que o percentual de mulheres $(94,8 \%)$ é maior que o de homens $(86,9 \%)$. 
TABELA 4. PORCENTAGEM DE HOMENS E MULHERES QUE VIVEM NAZONA URBANA CONDICIONAL A DOENÇA TUBERCULOSE

\begin{tabular}{lccc}
\hline \multirow{2}{*}{ Sexo } & $\begin{array}{c}\text { Pessoas que vivem na } \\
\text { zona urbana }(\%)\end{array}$ & $\begin{array}{c}\text { Pessoas sem tuberculose } \\
(\%)\end{array}$ & $\begin{array}{c}\text { Pessoas com tuberculose } \\
(\%)\end{array}$ \\
\hline Masculino & 84,6 & 84,6 & 86,9 \\
Feminino & 86,6 & 86,6 & 94,8 \\
\hline
\end{tabular}

FONTE: elaborado pelos autores com base nos dados da PNAD (2003)

Quando se considera características individuais, como cor do indivíduo, conforme a Tabela 5 , percebe-se que para os indivíduos que não contraíram tuberculose, a quantidade de homens é superior ao número de mulheres, para os indivíduos de cor branca. Porém, para os indivíduos de cor parda e negra, observa-se que a percentagem de homens supera àquela apresentada pelas mulheres.

TABELA 5. COR CONDICIONADA A DOENÇA TUBERCULOSE

\begin{tabular}{lccc}
\hline Variáveis & Gênero & $\begin{array}{c}\text { Pessoas sem } \\
\text { tuberculose (\%) }\end{array}$ & $\begin{array}{c}\text { Pessoas com } \\
\text { tuberculose (\%) }\end{array}$ \\
\hline \multirow{2}{*}{ Branca } & Masculino & 46,69 & 39,69 \\
& Feminino & 48,92 & 43,88 \\
\multirow{2}{*}{ Parda } & Masculino & 46,26 & 46,46 \\
& Feminino & 44,34 & 42,44 \\
\multirow{2}{*}{ Negra } & Masculino & 6,49 & 12,61 \\
& Feminino & 6,12 & 12,23 \\
\hline
\end{tabular}

FONTE: elaborado pelos autores com base nos dados da PNAD (2003)

Porém, quando se considera os indivíduos com tuberculose, a incidência é maior entre os indivíduos do sexo masculino e de cor parda e negra, enquanto que para os indivíduos do sexo feminino a percentagem concentra-se na cor branca.

Em termos de distribuição geográfica, de acordo com Tabela 6, para indivíduos não infectados pela tuberculose, percebe-se que nas regiões Norte e Centro-Oeste há um predomínio de homens em relação as mulheres. Porém, nas regiões Nordeste, Sudeste e Sul o número de mulheres supera o número de homens.

TABELA 6. REGIÕES CONDICIONADA A DOENÇA TUBERCULOSE

\begin{tabular}{lccc}
\hline Variáveis & Gênero & $\begin{array}{c}\text { Pessoas sem tuberculose } \\
(\%)\end{array}$ & $\begin{array}{c}\text { Pessoas com tuberculose } \\
(\%)\end{array}$ \\
\hline \multirow{2}{*}{ Norte } & Masculino & 9,77 & 9,84 \\
& Feminino & 9,65 & 11,87 \\
Nordeste & Masculino & 32,50 & 37,23 \\
& Feminino & 32,59 & 29,50 \\
Sudeste & Masculino & 29,48 & 28,00 \\
& Feminino & 29,76 & 30,21 \\
Sul & Masculino & 15,67 & 17,85 \\
& Feminino & 15,70 & 18,34 \\
Centro-Oeste & Masculino & 12,58 & 7,08 \\
& Feminino & 12,29 & 10,07 \\
\hline
\end{tabular}

FONTE: elaborado pelos autores com base nos dados da PNAD (2003)

Porém, quando se considera indivíduos contaminados pela tuberculose, como pode ser visto na Tabela 6, observa-se que nas regiões Norte, Sudeste, Sul e 
Centro-Oeste o número de mulheres supera o número de homens. Contudo, a região Nordeste é a que apresenta uma superioridade de casos em relação ao número de mulheres.

\section{Resultados e Discussão}

Os resultados das equações de tuberculose para homens e mulheres são apresentados na tabela 7. Essa tabela contém as variáveis, os efeitos marginais e a estatística z, que são essenciais para a análise.

Em primeiro lugar, apesar das estimações serem realizadas separadamente, os resultados confirmam o que é quase consenso na literatura: homens são mais afetados pela tuberculose do que as mulheres. Por se tratar de uma doença infecto-contagiosa, a proliferação da tuberculose aumenta de forma significativa em ambientes fechados como presídios, locais de trabalho, e domicílios com poucos cômodos. Como o percentual de homens que habita os presídios, que trabalha e que mora em domicílios subnormais é maior que o de mulheres, esta pode ser uma boa explicação para tal resultado.

A literatura define ainda que a incidência de tuberculose é maior entre indivíduos mais velhos e negros, como relatado nos trabalhos de Bock, McGowan Blumberg (1998); e Martinez, Small, e Behr (2000). Os resultados obtidos no presente estudo vão ao encontro dessa definição, tanto para homens como para mulheres.

As estimativas demonstram que para a variável idade a incidência de tuberculose é maior entre os idosos quando comparados às crianças e aos adultos. Ao se analisar os efeitos marginais, conclui-se que se o indivíduo é idoso, sua probabilidade de contrair a doença, em relação às crianças, aumenta em 0,0111 para homens, e em 0,0064 para as mulheres.

Para conhecer os efeitos líquidos da idade sobre a probabilidade de ser infectado pela tuberculose, é necessário somar os coeficientes estimados de adultos e idosos, pois o efeito é cumulativo. Nesse sentido, ser idoso aumenta o risco de contrair tuberculose em o,0130 para homens e em o,0081 para mulheres, tendo como variável base as crianças. Cabe ressaltar que o efeito mais forte é sobre os homens idosos.

Analisando-se a cor, conclui-se que os indivíduos de cor preta apresentam maior probabilidade de serem vítimas da tuberculose. Portanto, se o indivíduo é da cor branca e parda, o risco de se contrair a doença é reduzido em relação aos indivíduos de cor preta. Essa redução é, respectivamente, da ordem de 0,0008 e 0,0006 para homens; e de 0,0007 e 0,0006 para mulheres.

A incidência de tuberculose também varia conforme a localização do domicílio. Nesse sentido, residir na zona urbana ou na zona rural pode aumentar ou diminuir a probabilidade de se contrair a doença. Os resultados desse 
trabalho definem que viver no meio urbano eleva o risco de infecção pela tuberculose para ambos os sexos, sendo que o efeito maior é sobre as mulheres (o,0008). Esse resultado confirma a hipótese levantada pelos trabalhos de Tupasi et al. (2000) e pelo "Ministry of Health and Social Affairs, Korean Institute of Tuberculosis". Nestes estudos, o que explica a maior incidência de tuberculose no meio urbano é a intensa aglomeração de indivíduos vindos do meio rural nos arredores das grandes metrópoles, agravando a pobreza e o risco da doença.

Verifica-se na literatura que a incidência de tuberculose está relacionada com as condições de vida verificadas em determinado ambiente. Como elucidado nos trabalhos de Kuemmerer e Comstock (1967), Vicentin, Santo e Carvalho (2002), e Santos et al. (2007); o baixo nível sócio-econômico tende a elevar a probabilidade de se contrair a doença. Nesse caso, particularmente, a sobrelotação das habitações e as más condições sanitárias tendem a perpetuar a tuberculose entre os moradores da mesma casa e da vizinhança.

TABELA 7. RESULTADOS DAS EQUAÇÕES PROBIT PARA HOMENS E MULHERES ACOMETIDOS PELA TUBERCULOSE

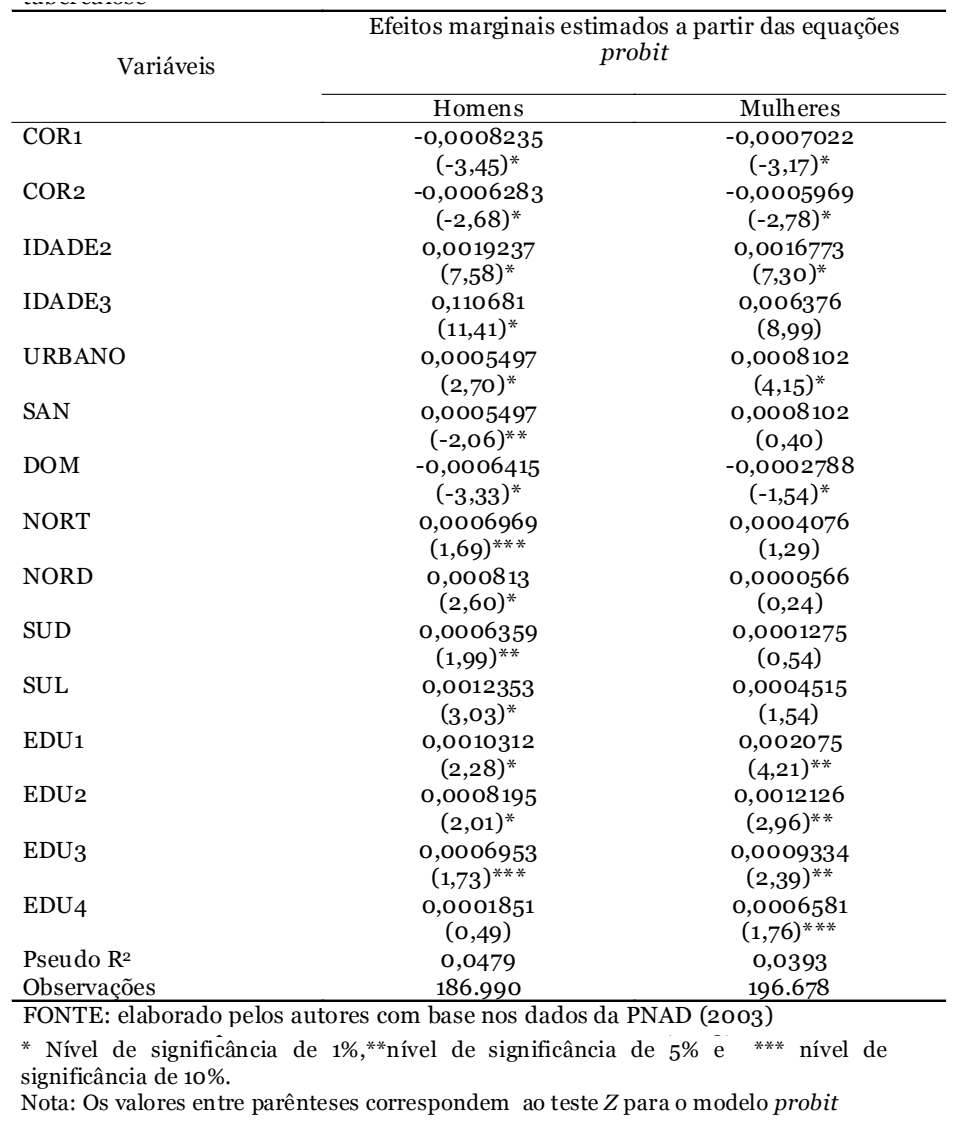


Os resultados obtidos nesse estudo confirmam tal hipótese para os homens. Se uma habitação não possui banheiro ou sanitário, menor é a higiene e maiores são as chances de se contrair tuberculose. Portanto, viver em uma habitação com banheiro ou sanitário diminui a probabilidade de se contrair a doença em 0,0007.

Por sua vez, quanto menor a quantidade de cômodos verificada em uma moradia, maior o risco de se contrair tuberculose, já que haverá mais contato entre os indivíduos. Nesse caso, ter mais cômodos em casa diminui o risco de ser vítima da doença em 0,0006 . Com relação às mulheres, não existe relação entre más condições de vida e incidência de tuberculose.

A localização geográfica de um indivíduo dentro do território brasileiro também impacta na probabilidade de ser infectado pela doença. Para os homens, morar nas regiões Sul, Sudeste, Norte e Nordeste, eleva o risco de se contrair tuberculose em relação ao centro-oeste. Nesse caso, a probabilidade de ser vitimizado pela tuberculose aumenta mais se o individuo mora no Sul do Brasil: o,0012. No caso das mulheres, não foi encontrada relação entre o fato de se residir em uma região específica do país e a incidência da doença.

A probabilidade de se contrair tuberculose é interligada com o nível educacional da população em estudo. Geralmente, indivíduos com mais anos de estudo tendem a apresentar um nível de renda mais elevado, o que diminui o risco de se contrair a doença. Além disso, um indivíduo com maior nível de instrução possui, geralmente, maior conhecimento sobre as formas de propagação de determinadas doenças e, portanto, pode se prevenir melhor, a fim de correr menos risco de vitimização.

Essa relação entre o nível educacional e o risco de ser contaminado pela tuberculose é confirmada nesse trabalho, tanto para os homens como para as mulheres, o que vai ao encontro dos resultados obtidos por Kuemmerer e Comstock (1967); e Santos et al. (2007). No caso dos homens, se um indivíduo possui o menor nível de educação (o a 4 anos de estudo), a probabilidade de se contrair a tuberculose é elevada em 0,0010. Entre as mulheres, tal elevação é da ordem de 0,0021.

Mas, para que o impacto da educação sobre a incidência de tuberculose seja analisado de forma precisa, é necessário somar os efeitos marginais das faixas de anos de estudo definidas nesse trabalho, a fim de que seja obtido o efeito líquido sobre o risco de se contrair a doença. Nesse sentido, para os homens, o menor nível educacional eleva o risco de incidência da tuberculose em 0,0027. Para as mulheres, esse efeito é quase duas vezes superior: 0,0049. 


\section{Considerações finais}

O objetivo do trabalho foi analisar os determinantes socio econômicos da tuberculose, com ênfase na educação e nas condições de vida da população brasileira. A tuberculose, enfermidade considerada por muitos como uma "doença do passado", vem aumentando consideravelmente em todo o mundo, principalmente ns regiões mais pobres. O Brasil está entre os países mais infectados pela tuberculose, dada a péssima condição de vida de boa parte da população. Nesse sentido, é extremamente relevante trabalhos que estabeleçam relações entre características socio econômicas da população e a incidência da doença.

Os resultados do presente estudo demonstram que características individuais como cor e idade são importantes na probabilidade de se contrair a doença. Nesse sentido, percebe-se que se o indivíduo for idoso, homem e de cor preta a probabilidade de se contrair a doença é mais elevada.

De uma forma geral, percebe-se que a probabilidade de um indivíduo ser acometido pela tuberculose é mais elevada caso ele resida em uma zona urbana. Esse resultado pode ser explicado pela concentração da população pobre nas grandes cidades do país. Essa parcela da população vive em péssimas condições de vida, dadas a insuficiência de condições sanitárias e a aglomeração de indivíduos numa mesma moradia. A partir dos resultados desse trabalho, ficou comprovado que se um indivíduo de ambos os gêneros vive em uma habitação com banheiro ou sanitário, e com um número maior de cômodos, há uma diminuição no risco de incidência da doença.

Em relação a localização geográfica dos homens, os resultados demonstram que residir nas regiões Norte, Nordeste, Sul e Sudeste eleva a probabilidade de se contrair tuberculose em relação à região Centro-Oeste do país. Cabe sublinhar que morar na região Sul corresponde ao maior risco observado de infecção dentre todas as regiões brasileiras. No caso das mulheres, não existe relação entre a probabilidade de ser contaminado pela tuberculose e alguma região específica do Brasil.

A educação é uma variável extremamente relevante na determinação do risco de incidência da tuberculose, dada a sua correlação direta com o nível de renda e com o nível de informação sobre formas de prevenção da doença. Os resultados do trabalho, no geral, apontam que um indivíduo com menor nível educacional apresenta maior probabilidade de contrair a doença. Por outro lado, ao se comparar baixos níveis de educação (o a 4 anos de estudo) entre os gêneros, percebe-se que o efeito sobre as mulheres é quase duas vezes maior. Nesse caso, o risco de uma mulher com baixo nível educacional ser infectada pela tuberculose é bem mais elevado quando comparado com um homem com o mesmo nível de instrução. 
Existe um consenso de que a educação é a variável chave para a melhoria das condições socio econômicas. Nesse sentido, propõe-se a formulação de políticas públicas, a fim de elevar o nível de instrução da população e consequentemente diminuir o risco de proliferação de doenças relacionas às baixas condições de vida como a tuberculose.

\section{Referências}

BERGNER, L. \& YERBY, A.S. (1968). "Low income and barriers to use of health services”. New England Journal of Medicine. 278: 541-6.

BOCK, N. N. \& MCGOWAN, J. E. \& BLUMBERG, H. M. (1998). "Few opportunities found for tuberculosis prevention among the urban poor". The International Journal of Tuberculosis and Lung Disease. 2: 124-129.

CANTWELL, M. F. \& SNIDER D. E. Jr. \& CAUTHEN. G. M. \& ONORATO. I. M. (1994). "Epidemiology of tuberculosis in the United States, 1985 through 1992 ". Journal of the American Medical Association. 272: 535-9.

D'ARCY HART P.(1932). “The value of tuberculin tests in man, with special reference to the intracutaneous test”. Med. Res. Council Special Series. 164: 5-132.

ENARSON, D. A. \& WANG. J. S. \& DIRKS. J. M. (1989). "The incidence of active tuberculosis in a large urban area”. American Journal Epidemiology, 129: 1268-76.

GROTH-PETERSEN. E. \& KNUDSEN. J. \& WILBEK. E. (1959). “Epidemiological basis of tuberculosis eradication inan advanced country". Bull. World Health Organ, 21: 5-49.

HOLMES, C. B. \& HAUSLER, H. \& NUNN. P. (1998). “A review of sex differences in the epidemiology of tuberculosis". The International Journal Tuberculosis and Lung Disease. 2: 96-104.

KUEMMERER. J. M. \& COMSTOCK. G. W. (1967). “Sociologic concomitants of tuberculin sensitivity”. American Review Respiratory Disease. 96: 885-92.

MARTINEZ, A. N. \& J. T. RHEE \& P. M. SMALL \& M. A. BEHR. (2000). "Sex differences in the epidemiology of tuberculosis in San Francisco". The International Journal of Tuberculosis and Lung Disease. 4:26-31

MINISTRY OF HEALTH AND SOCIAL AFFAIRS, KOREAN INSTITUTE OF TUBERCULOSIS, KOREAN NATIONAL TUBERCULOSIS ASSOCIATION.(1966). Report on the first tuberculosis prevalence survey in Korea - 1965. Edition 1. Seoul: The Korean Institute of Tuberculosis.

NATIONAL TUBERCULOSIS INSTITUTE BANGALORE. (1974). “Tuberculosis in a rural population of South India: a five-year epidemiological study”. Bull. World Health Organ. 51: 473-88.

RIEDER, H. L. (2001). Bases epidemiológicas do controle da tuberculose. Lisboa: Direção-Geral da Saúde. p. 168. 
SANTOS, Maria de Lourdes Sperli Geraldes dos \& VENDRAMINII, Silvia Helena Figueiredo \& GAZETTA, Cláudia Eli \& OLIVEIRA, Sonia Aparecida da Cruz 7 VILLA, Tereza Cristina Scatena. (2007). "Poverty:socioeconomic characterization at tuberculosis". Revista Latino-Americana de Enfermagem. 15: 762-767.

TUPASI, T. E \& RADHAKRISHNA, S \& QUELAPIO, M. I. D. (2000). “Tuberculosis in the urban poor settlements in the Philippines". The International Journal of Tuberculosis and Lung Disease. 4: 4-11.

VICENTIN, G. \& SANTO, A. H. \& CARVALHO, M. S. (2002). "Mortalide por Tuberculose e Indicadores Sociais no Município do Riode Janeiro". Ciência \& Saúde Coletiva. 7(2): 63-81.

Recebido em: 15 de setembro de 2010

Primeira resposta em: 23 de novembro de 2010

Aceite em: 14 de dezembro de 2010 